\title{
High SHIP2 Expression Indicates Poor Survival in Colorectal Cancer
}

\author{
Ju Yang, ${ }^{1}$ Maoying Fu, ${ }^{2}$ Yaoguang Ding, ${ }^{3}$ Yajing Weng, ${ }^{4}$ Weifei Fan, ${ }^{5}$ \\ Xiaolin Pu, ${ }^{5}$ Zhijun Ge, ${ }^{6}$ Feng Zhan, ${ }^{7}$ Huihui Ni, ${ }^{2}$ Wei Zhang, ${ }^{2}$ Feng Jin, \\ Ning Xu, ${ }^{8}$ Jiang Li, ${ }^{9}$ Liang Qiu, ${ }^{9}$ Jun Wang, and Xuefeng $\mathbf{G u}^{2}$ \\ ${ }^{1}$ Department of Gastroenterology, Kunshan Hospital of Traditional Chinese Medicine, \\ The Affiliated Hospital of Nanjing University of Chinese Medicine, Suzhou 215000, China \\ ${ }^{2}$ Department of Infectious Diseases, The First People's Hospital of Kunshan Affiliated with Jiangsu University, Suzhou 215000, China \\ ${ }^{3}$ Department of Oncology, Kunshan Hospital of Traditional Chinese Medicine, \\ The Affiliated Hospital of Nanjing University of Chinese Medicine, Suzhou 215000, China \\ ${ }^{4}$ Department of Geriatric Rehabilitation, Kunshan Hospital of Traditional Chinese Medicine, \\ The Affiliated Hospital of Nanjing University of Chinese Medicine, Suzhou 215000, China \\ ${ }^{5}$ Department of Hematology and Oncology, Jiangsu Province Geriatric Institute, Nanjing 210000, China \\ ${ }^{6}$ Department of Anesthesiology, Yixing People's Hospital, The Affiliated Yixing Hospital of Jiangsu University, Yixing 214200, China \\ ${ }^{7}$ Department of Hepatobiliary and Laparoscopic Surgery, Yixing People's Hospital, The Affiliated Yixing Hospital of Jiangsu University, \\ Yixing 214200, China \\ ${ }^{8}$ Department of Pathology, Nanjing Medical University, Nanjing 210000, China \\ ${ }^{9}$ Department of Pathology, Jiangsu Province Geriatric Institute, Nanjing 210000, China
}

Correspondence should be addressed to Jun Wang; wangjjs2013@163.com and Xuefeng Gu; sani666@163.com

Received 3 August 2014; Revised 23 October 2014; Accepted 1 November 2014; Published 24 November 2014

Academic Editor: Chao Hung Hung

Copyright (C) $2014 \mathrm{Ju}$ Yang et al. This is an open access article distributed under the Creative Commons Attribution License, which permits unrestricted use, distribution, and reproduction in any medium, provided the original work is properly cited.

SH2-containing inositol $5^{\prime}$-phosphatase 2 (SHIP2), which generally regulates insulin signaling, cytoskeleton remodeling, and receptor endocytosis, has been suggested to play a significant role in tumor development and progression. However, the associations between SHIP2 expression and the clinical features to evaluate its clinicopathologic significance in colorectal cancer (CRC) have not been determined yet. In the present study, one-step quantitative real-time polymerase chain reaction (qPCR) test and immunohistochemistry (IHC) analysis with CRC tissue microarrays (TMA) were employed to evaluate the mRNA and protein expression of SHIP2 in CRC. The results showed that SHIP2 expression in the mRNA and protein levels was significantly higher in CRC tissues than that in corresponding noncancerous tissues (both $P<0.05$ ). The expression of SHIP2 protein in CRC was related to lymph node metastasis $(P=0.036)$, distant metastasis $(P=0.001)$, and overall survival $(P=0.009)$. Kaplan-Meier method and Cox multifactor analysis suggested that high SHIP2 protein level $(P=0.040)$ and positive distant metastasis $(P=0.048)$ were critically associated with the unfavorable survival of CRC patients. The findings suggested that SHIP2 may be identified as a useful prognostic marker in CRC and targeting CRC may provide novel strategy for CRC treatment.

\section{Introduction}

Colorectal cancer (CRC) is the third most common malignancy in the world, with an estimated incidence of more than 1.2 million new cases and over 600000 deaths globally $[1-3]$. The number of patients who are suffering continues to rise, especially in most Asian countries [4]. During the last two decades, several countries including China, South Korea, and Japan have witnessed a two- to threefold rise in incidence of CRC. In China, for example, the total number of CRC cases increased by $19.1 \%$ and $17.7 \%$ in Chinese males and females from 2000 to 2005 , respectively $[5,6]$. Generally speaking, ulcerative colitis, familial adenomatous polyposis, and hereditary nonpolyposis colon cancer syndrome are 
the three major risks that contribute to the CRC development [7]. Despite the development of combined therapeutic modalities for CRC treatment, including surgical operation and combination of chemotherapy and adjuvant therapy, the overall prognosis of CRC remains unsatisfactory and the 5-year survival rate of CRC patients with metastasis is still under $10 \%[8,9]$. It is valuable and critical to discover molecular predictive markers for the prognosis, which would optimize the selection of therapeutic strategies and further improve patients' survival for CRC [10].

SH2-containing inositol $5^{\prime}$-phosphatase 2 (SHIP2) belongs to the phosphoinositol phosphatases family which plays important role in modulating signaling pathways relevant to both diabetes and cancer and generally regulates insulin signaling, cytoskeleton remodeling, and receptor endocytosis [11-13]. As one of the important lipid phosphatases that act downstream of phosphoinositide- $3^{\prime}$ kinase (PI3K), SHIP2 converts phosphatidylinositol (3,4,5)-trisphosphate (PIP3) into phosphatidylinositol $(3,4)$-biphosphate (PIP2) and subsequently reduces the activation of PI3K/Akt signaling cascade, which exerts energetic function in tumor development and progression [14-16]. Under this circumstance, SHIP2 is supposed to show anticancer effects and several studies support this presumption $[17,18]$. However, on the contrary, several studies elucidate the prooncogenic function of SHIP2 in certain type of cancer. For instance, high SHIP2 expression enhances cancer cell proliferation, while inhibition of SHIP2 suppresses cancer growth in vitro and distant metastases in vivo [12]. Similarly, SHIP2 performs oncogenically and high expression of SHIP2 indicates poor survival of breast cancer and laryngeal squamous cell carcinoma $[19,20]$. SHIP2 also promotes cancer cell proliferation and metastasis by preventing epidermal growth factor receptor (EGFR) turnover and enhancing EGF-induced Akt activation [12, 21]. In light of the dual characteristics of SHIP2, the association of SHIP2 expression and cancer development still seems vague and little is known about the role of SHIP2 in CRC, especially the prognostic significance.

In this present paper, we detected SHIP2 expression in a number of CRC samples and analyzed the associations between SHIP2 expression and clinicopathologic items in CRC patients. Finally, the prognostic significance of SHIP2 was evaluated in 102 CRC cases.

\section{Materials and Methods}

2.1. Patients and Tissue Samples. A total of 15 fresh CRC tissues and matched tumor-adjacent noncancerous tissues were collected from the tissue bank of the Affiliated Hospital of Nantong University to perform quantitative real-time polymerase chain reaction (qPCR) test. 102 formalin-fixed, paraffin-embedded CRC tissues and corresponding tumoradjacent normal tissues were also enrolled in this present study from the Affiliated Hospital of Nantong University from 2002 to 2005 to execute immunohistochemistry (IHC) analysis. Other pieces of clinicopathological information of 102 CRC cases, such as gender, age, tumor size, tumor location, histological type, tumor differentiation, serum CEA level, metastasis status, TNM stage, and overall survival, were collected from each patient's medical records simultaneously. Clinical staging was performed according to the latest revision of American Joint Committee on Cancer/International Union Against Cancer TNM staging system [22].

\subsection{Detection of the SHIP2 Expression by One-Step qPCR Test.} Total RNA was extracted from 15 cases of the frozen CRC tissues and the matched noncancerous tissues using the Trizol reagent (Invitrogen, Carlsbad, CA, USA) according to the manufacturer's guidelines. Total RNA extraction and onestep qPCR analysis were performed as previously described [23]. The primers for SHIP2 were as follows: forward primer $5^{\prime}$-TCG TCA CCA GCG ACC ATT C-3' and reverse primer $5^{\prime}$-AGC CCT TTC TTG GAG ATG AAC TG-3'. The glyceraldehyde 3-phosphate dehydrogenase (GAPDH) mRNA level was used to standardize the measurements of SHIP2 and the primers for GAPDH were as follows: forward primer $5^{\prime}$-TGC ACC ACC AAC TGC TTA GC- $3^{\prime}$ and reverse primer $3^{\prime}-\mathrm{GGC}$ ATG GAC TGT GGT CAT GAG-5'.

2.3. Detection of the SHIP2 Expression by IHC Analysis. Tissue microarray (TMA) was produced by Alenabio Biotech (Xi'an, China). Core tissue biopsies ( $2 \mathrm{~mm}$ in diameter) were collected from individual paraffin-embedded CRC sections and arranged in the recipient paraffin blocks. IHC analysis was performed as previously described [24]. Tissue sections were incubated with polyclonal goat anti-SHIP2 antibody (Santa Cruz Biotechnology, Santa Cruz, CA, USA) in TBS containing 1\% bovine serum albumin for 1 hour. The secondary antibody used was horseradish peroxidaseconjugated anti-goat antibody (Dako Cytomation, Carpinteria, CA, USA).

SHIP2 immunostaining was scored by two independent pathologists according to intensity and percentage of positive cells simultaneously. Staining intensity was scored as follows: 0 (negative), 1 (weakly positive), 2 (moderately positive), and 3 (strongly positive). The percentage of SHIP2-positive cells was also scored according to four categories as follows: 1 was given for $0 \%$ to $10 \%, 2$ for $11 \%$ to $50 \%$, 3 for $51 \%$ to $80 \%$, and 4 for $81 \%$ to $100 \%$. The product of the intensity and percentage scores was used as the final staining score. The degree of SHIP2 staining was quantified using a two-level grading system as follows: samples with a sum score $<4$ were considered as low SHIP2 expression while those with a sum score $\geq 4$ were considered as high SHIP2 expression.

2.4. Statistical Analysis. Statistical analyses were performed by using SPSS 16.0 (SPSS Inc., Chicago, IL, USA) and STATA 12.0 (Stata Corporation, College Station, TX, USA). The expression of SHIP2 mRNA in fresh frozen CRC tissues and matched tumor-adjacent normal tissues normalized to GAPDH was analyzed with the Wilcoxon nonparametric signed-rank test. The relationship between SHIP2 protein expression and clinicopathological factors was evaluated by Chi-square test. Univariate and multivariate analyses were performed using Cox's proportional hazards regression models. Survival curves were calculated using the Kaplan-Meier 


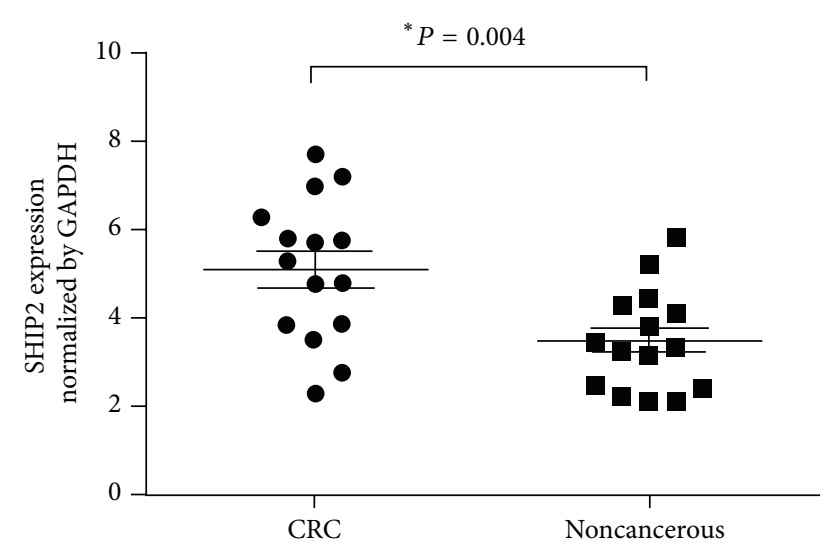

FIGURE 1: One-step quantitative real-time polymerase chain reaction (qPCR) test was employed to evaluate the expression of SHIP2 mRNA in colorectal cancer (CRC) and tumor-adjacent noncancerous tissues. When normalized to GAPDH, the expression of SHIP2 mRNA in CRC tissues $(5.11 \pm 0.419)$ was significantly higher than that in matched noncancerous tissues $(3.48 \pm 0.295)(P=0.004)$.

method. For all tests, the significance level for statistical analysis was set at $P<0.05$.

\section{Results}

3.1. Summarization of the Characteristics of 102 CRC Patients. A total of 102 CRC cases were enrolled from 63 men and 39 women in this present study. The mean age of all patients at the time of diagnosis was $63.08 \pm 10.852$ years. The tumor diameters of 44 cases were $\geq 5 \mathrm{~cm}$ and of 58 cases were $<5 \mathrm{~cm}$. The tumors of 87 cases were located in colon, 11 cases in rectum, and 4 cases in ileocecal junction. The histological type of tumor in 99 cases was adenocarcinoma, whereas the other 3 cases were identified as nonadenocarcinoma. Four patients suffered from a well-differentiated tumor, 82 had moderate tumor differentiation, and 7 had poor tumor differentiation. There were 11 cases with serum CEA level $\geq 15 \mathrm{ng} / \mathrm{mL}$ and 59 cases with serum CEA level $<15 \mathrm{ng} / \mathrm{mL}$. Positive lymph node metastasis was noticed in 34 patients while distant metastasis was observed in 19 patients. According to TNM staging system, 63 patients were in stages I-II and 39 patients were in stages III-IV. Of all the 102 cases, 61 patients survived while 41 patients died.

3.2. Evaluation of SHIP2 $m R N A$ Expression by qPCR Test. Total RNA was extracted from 15 CRC tissues and matched noncancerous tissues and subsequently subjected to onestep qPCR for the evaluation of the expression of SHIP2 mRNA. When normalized to GAPDH, the means of SHIP2 mRNA expression in CRC tissues and the corresponding noncancerous tissues were $5.11 \pm 0.419$ and $3.48 \pm 0.295$, respectively $(t=3.174, P=0.004)$. SHIP2 mRNA expression averaged 1.47-fold higher in CRC samples than that in noncancerous tissue samples (Figure 1).

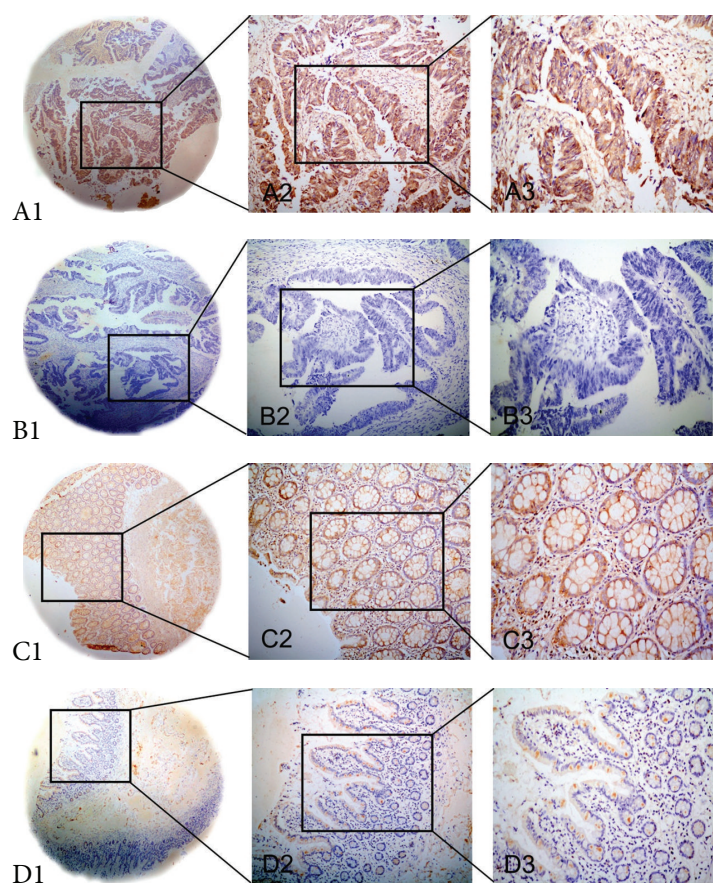

FIGURE 2: Representative images of SHIP2 protein expression in colorectal cancer (CRC) tissues and corresponding noncancerous tissues with tissue microarray (TMA). A1, A2, and A3: high immunohistochemical (IHC) staining of SHIP2 protein expression in CRC tissue samples. B1, B2, and B3: low IHC staining of SHIP2 protein expression in CRC tissue samples. $\mathrm{C} 1, \mathrm{C} 2$, and $\mathrm{C} 3$ : high IHC staining of SHIP2 protein expression in noncancerous tissue samples. D1, D2, and D3: low IHC staining of SHIP2 protein expression in noncancerous tissue samples. Original magnification $\times 40$ in $\mathrm{A} 1, \mathrm{~B} 1, \mathrm{C} 1$, and $\mathrm{D} 1 ; \times 200$ in $\mathrm{A} 2, \mathrm{~B} 2, \mathrm{C} 2$, and $\mathrm{D} 2 ; \times 400$ in $\mathrm{A} 3$, $\mathrm{B} 3, \mathrm{C} 3$, and $\mathrm{D} 3$.

3.3. Evaluation of SHIP2 Protein Expression by IHC Analysis. IHC analysis was further performed to detect the SHIP2 protein expression in CRC. High SHIP2 expression was detected in $51(50.0 \%)$ out of 102 CRC tissues and in 24 (23.5\%) out of 102 tumor-adjacent noncancerous tissues. The results exhibited significant difference $(P<0.05)$ and were consistent with results of SHIP2 mRNA expression in qPCR test. Positive staining was mainly localized in the cytoplasm of CRC cells. Typical IHC staining patterns for SHIP2 in CRC are shown in Figure 2.

3.4. Relationships between SHIP2 Protein Expression and Clinicopathological Items. The relationships between high SHIP2 protein expression and selected clinicopathological items were shown in Table 1. High SHIP2 protein expression was significantly related to lymph node metastasis $(P=$ $0.036)$, distant metastasis $(P=0.001)$, and overall survival $(P=0.009)$ (Table 1$)$.

3.5. Survival Analysis. In order to evaluate the prognostic role of SHIP2, survival analyses were subsequently executed. In univariate analysis, 4 items including high SHIP2 protein expression $(P=0.017)$, lymph node metastasis $(P=0.001)$, 
TABLE 1: The relationship between SHIP2 expression and clinical attributes of 102 CRC patients.

\begin{tabular}{|c|c|c|c|c|c|}
\hline \multirow{2}{*}{ Groups } & \multirow{2}{*}{ Number } & \multicolumn{2}{|c|}{ SHIP2 } & \multirow{2}{*}{$\chi^{2}$} & \multirow{2}{*}{$P$ value } \\
\hline & & + & $\%$ & & \\
\hline \multicolumn{6}{|l|}{ Gender } \\
\hline Male & 63 & 31 & 49.2 & \multirow{2}{*}{0.04} & \multirow{2}{*}{0.839} \\
\hline Female & 39 & 20 & 51.3 & & \\
\hline \multicolumn{6}{|l|}{ Age (years) } \\
\hline$\geq 60$ & 68 & 34 & 50.0 & \multirow{2}{*}{0.00} & \multirow{2}{*}{1.000} \\
\hline$<60$ & 34 & 17 & 50.0 & & \\
\hline \multicolumn{6}{|l|}{ Tumor size $(\mathrm{cm})$} \\
\hline$\geq 5$ & 44 & 21 & 47.7 & \multirow{2}{*}{0.16} & \multirow{2}{*}{0.689} \\
\hline$<5$ & 58 & 30 & 51.7 & & \\
\hline \multicolumn{6}{|l|}{ Tumor location } \\
\hline Colon & 87 & 43 & 49.4 & \multirow{3}{*}{1.10} & \multirow{3}{*}{0.576} \\
\hline Rectum & 11 & 5 & 45.5 & & \\
\hline Ileocecal junction & 4 & 3 & 75.0 & & \\
\hline \multicolumn{6}{|l|}{ Histological type } \\
\hline Adenocarcinoma & 99 & 48 & 48.5 & \multirow{2}{*}{3.09} & \multirow{2}{*}{0.079} \\
\hline Nonadenocarcinoma & 3 & 3 & 100.0 & & \\
\hline \multicolumn{6}{|l|}{ Tumor differentiation } \\
\hline Well & 4 & 1 & 25.0 & \multirow{4}{*}{1.32} & \multirow{4}{*}{0.518} \\
\hline Moderately & 82 & 44 & 53.7 & & \\
\hline Poorly & 7 & 4 & 57.1 & & \\
\hline Insufficient data & 9 & 2 & & & \\
\hline \multicolumn{6}{|l|}{ Serum CEA level (ng/mL) } \\
\hline$\geq 15$ & 11 & 6 & 54.5 & \multirow{3}{*}{0.30} & \multirow{3}{*}{0.581} \\
\hline$<15$ & 59 & 25 & 42.4 & & \\
\hline Insufficient data & 32 & 20 & & & \\
\hline \multicolumn{6}{|l|}{ Lymph node metastasis } \\
\hline Positive & 34 & 22 & 64.7 & 4.41 & $0.036^{*}$ \\
\hline Negative & 68 & 29 & 42.6 & & \\
\hline Distant metastasis & & & & & \\
\hline Positive & 19 & 17 & 89.5 & 1455 & $0001^{*}$ \\
\hline Negative & 83 & 34 & 41.0 & 14.00 & \\
\hline TNM stage & & & & & \\
\hline Stages I, II & 63 & 27 & 42.9 & 3.36 & 0.067 \\
\hline Stages III, IV & 39 & 24 & 61.5 & & \\
\hline Overall survival & & & & & \\
\hline Survival & 61 & 24 & 39.3 & 6.89 & $0.009^{*}$ \\
\hline Death & 41 & 27 & 65.9 & 0.0 & 0.00 \\
\hline
\end{tabular}

${ }^{*} P<0.05$.

distant metastasis $(P=0.001)$, and TNM stage $(P=0.001)$ showed a significant correlation with the overall survival rate of 102 CRC patients. Furthermore, multivariate analysis confirmed that high SHIP2 expression $(P=0.040)$ and distant metastasis $(P=0.001)$ were two independent prognostic factors for CRC (Table 2). Kaplan-Meier survival curves demonstrated that CRC patients with high SHIP2 expression and positive distant metastasis encountered a significantly poorer survival time (Figure 3 ).

\section{Discussion}

In insulin signaling, SHIP2 plays a substantial role in the negative regulation of insulin sensitivity [14]. SHIP2 hydrolyzes the PI3K product PIP3 to PIP2 and inhibits the performance of PI3K/Akt signaling simultaneously [25]. In turn, SHIP2 suppresses cell growth through regulating intracellular insulin sensitivity. On the other hand, SHIP2 also regulates cell adhesion pathways which take great effects in the movement of cells. Considering the fact that the SHIP2 may function diversely in adhesion signaling by comparing to its role in insulin signaling, it is highly possible that abnormal expression of SHIP2 in some pathological conditions may impart its capability on cancer development from insulin regulation in normal physiological situations [12]. Accordingly, a review by Suwa et al. states that SHIP2 shows both prooncogenic effects and anticancer effects for SHIP2 function may differ based on interactive and imbalanced modulation by upstream stimulators including insulin, EGF, and IGF-I and downstream molecules including Akt2, tyrosine kinase receptor, and focal adhesion adaptor proteins [26]. For CRC, a great number of studies pointed the crucial relationships in the expression of adhesion proteins and catenin pathway with CRC development [27-29]; the exact role of SHIP2 in CRC is barely explored.

In this study, the qPCR result showed that the SHIP2 mRNA expressions in CRC tissues were higher than those in normal noncancerous tissues. Furthermore, TMA with CRC samples was constructed and IHC analysis was performed to confirm that higher SHIP2 protein expression was detected in CRC samples than that in matched noncancerous samples. The data elucidated the oncogenic role of SHIP2 in tumorigenesis in CRC and were consistent with previous studies that indicated high expression of SHIP2 in cancer tissues [19, 20]. Besides, high SHIP2 expression in CRC was correlated with several clinical attributes, including lymph node metastasis, distant metastasis, and overall survival. The results showing the prooncogenic effect of SHIP2 were in accord with our latest study concerning SHIP2 expression in lung cancer [23]. Similarly, a number of mechanical speculations have been raised to explore the characteristics of SHIP2 in cancer development. SHIP2 may regulate cell spreading and induce generation of local pools of PIP2, which play critical parts in the actin remodeling involved in the movement of cells [30]. SHIP2 may perform as a scaffolding protein to promote specific interaction with substantial cytoskeleton regulators, thereby subsequently controlling actin remodeling $[26,31]$. To summarize, SHIP2 is believed to modulate the ability of cells to migrate and attach via maintenance and remodeling of the actin cytoskeleton. Hence the loss of focal adhesion leading to inhibitory spreading ability indicates defective cell locomotion, which in turn regulates cancer cell migration and invasion during metastasis. Our data of the associations between SHIP2 expression and clinicopathologic items in CRC in this present study were also in agreement with the above reports concerning the prooncogenic effects of SHIP2.

In addition, high SHIP2 protein expression, lymph node metastasis, distant metastasis, and TNM stage were revealed to be correlated with overall survival of CRC patients by 
TABLE 2: Univariate and multivariate analyses of prognostic factors in 102 CRC patients for overall survival.

\begin{tabular}{|c|c|c|c|c|c|c|}
\hline & \multicolumn{3}{|c|}{ Univariate analysis } & \multicolumn{3}{|c|}{ Multivariate analysis } \\
\hline & HR & $P>|z|$ & $95 \% \mathrm{CI}$ & HR & $P>|z|$ & $95 \% \mathrm{CI}$ \\
\hline \multicolumn{7}{|l|}{ SHIP2 expression } \\
\hline High versus low & 2.20 & $0.017^{*}$ & $1.151-4.189$ & 2.05 & $0.040^{*}$ & $1.031-3.820$ \\
\hline \multicolumn{7}{|l|}{ Gender } \\
\hline Male versus female & 1.44 & 0.281 & $0.744-2.774$ & & & \\
\hline \multicolumn{7}{|l|}{ Age (years) } \\
\hline$\geq 60$ versus $<60$ & 0.83 & 0.562 & $0.439-1.565$ & & & \\
\hline \multicolumn{7}{|l|}{ Tumor size $(\mathrm{cm})$} \\
\hline$\geq 5$ versus $<5$ & 1.29 & 0.410 & $0.701-2.388$ & & & \\
\hline \multicolumn{7}{|l|}{ Tumor location } \\
\hline Colon versus rectum versus ileocecal junction & 0.68 & 0.354 & $0.306-1.528$ & & & \\
\hline \multicolumn{7}{|l|}{ Histological type } \\
\hline Adenocarcinoma versus nonadenocarcinoma & 1.05 & 0.960 & $0.145-7.662$ & & & \\
\hline \multicolumn{7}{|l|}{ Tumor differentiation } \\
\hline Well and moderately versus poorly & 1.50 & 0.413 & $0.569-3.943$ & & & \\
\hline \multicolumn{7}{|l|}{ Serum CEA level (ng/mL) } \\
\hline$\geq 15$ versus $<15$ & 2.33 & 0.057 & $0.976-5.573$ & & & \\
\hline \multicolumn{7}{|l|}{ Lymph node metastasis } \\
\hline Positive versus negative & 2.91 & $0.001^{*}$ & $1.573-5.408$ & 1.26 & 0.668 & $0.443-3.568$ \\
\hline \multicolumn{7}{|l|}{ Distant metastasis } \\
\hline Positive versus negative & 7.06 & $0.001^{*}$ & $3.687-13.521$ & 5.09 & $0.001^{*}$ & $2.034-12.736$ \\
\hline \multicolumn{7}{|l|}{ TNM stage } \\
\hline Stages I-II versus stages III-IV & 0.25 & $0.001^{*}$ & $0.129-0.466$ & 0.72 & 0.638 & $0.188-2.789$ \\
\hline
\end{tabular}

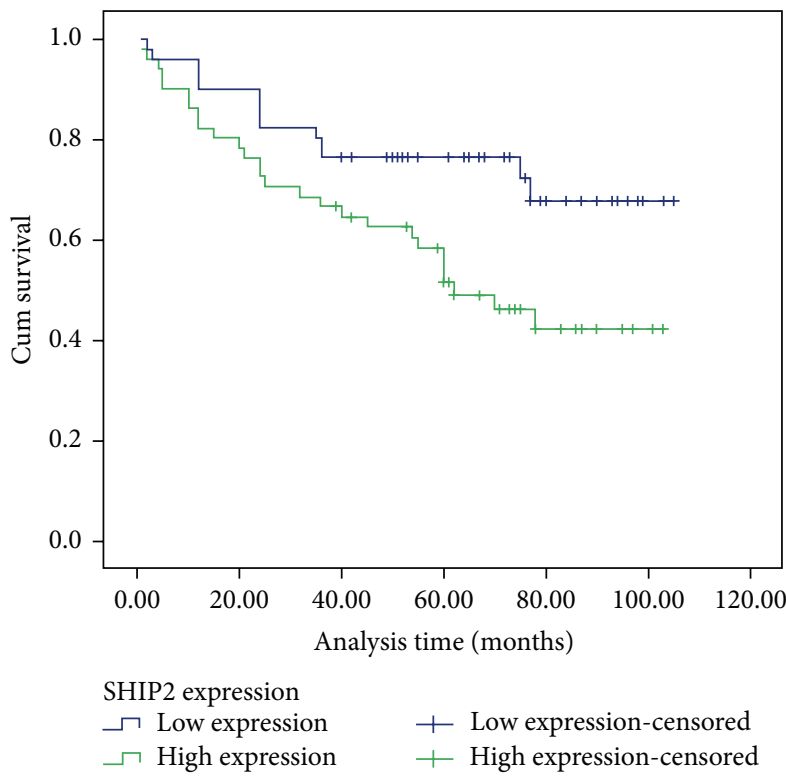

(a) Kaplan-Meier survival estimates, by SHIP2 expression

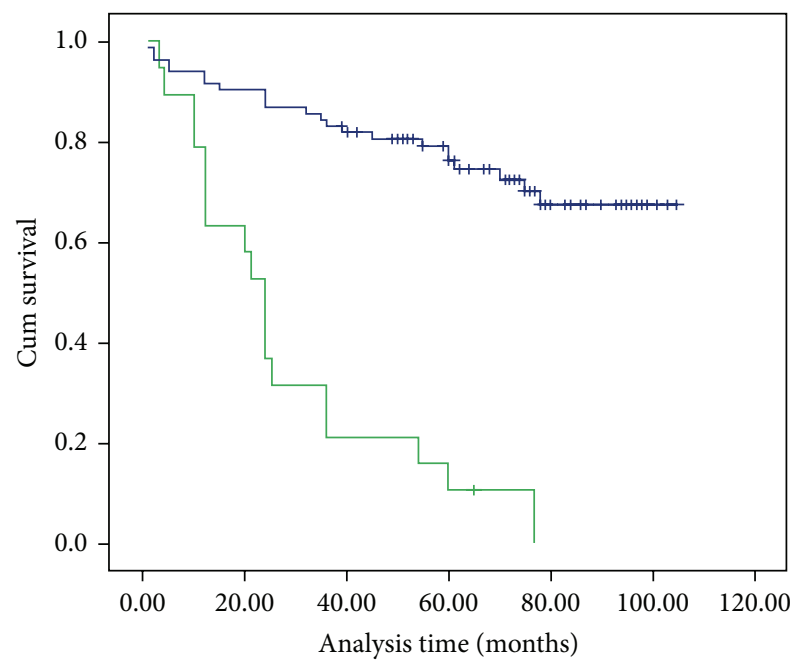

Distant metastasis

$\neg$ Negative distant metastasis + Negative distant metastasiscensored

$\neg$ Positive distant metastasis + Positive distant metastasiscensored

(b) Kaplan-Meier survival estimates, by distant metastasis

FIGURE 3: Survival analysis of colorectal cancer (CRC) patients by Kaplan-Meier method. (a) Overall survival rate in patients with high SHIP2 protein expression (green line) was significantly lower than that in patients with low SHIP2 expression (blue line). (b) Overall survival rate in patients with positive distant metastasis (green line) was significantly lower than that in patients with negative distant metastasis (blue line). 
univariate analysis while high SHIP2 expression and distant metastasis were further authenticated as independent predictors for unfavorable overall survival of CRC by multivariate analysis. Kaplan-Meier analysis finally demonstrated that the life span of patients with high SHIP2 expression was longer than that of patients with negative expression. Several studies, including two researches of ourselves, support our data announcing the prognostic role of SHIP2 in cancer $[19,20,23,24]$. Hence, it is rational to presume that SHIP2 plays oncogenic role dominantly in CRC based on this present research. Detailed studies of PI3K/Akt signaling and adhesion protein interacting with SHIP2 in various cell types or tissues are necessary to clarify the potential role of SHIP2 in procarcinogenesis.

In conclusion, we identified for the first time that a higher expression of SHIP2 in CRC tissues compared to the noncancerous tissues and SHIP2 might play an essential role as a prognostic marker of survival in patients with CRC. Our study is helpful in understanding the characteristics of SHIP2 in CRC development.

\section{Ethical Approval}

The study protocol was approved by the Ethics Committee of Nanjing Medical University and each local hospital. Written informed consent was acquired from all of the patients that were enrolled in this paper.

\section{Conflict of Interests}

The authors declare no conflict of interests.

\section{Authors' Contribution}

Jun Wang and Xuefeng Gu conceived and designed the experiments. Yaoguang Ding, Yajing Weng, Huihui Ni, Wei Zhang, Feng Jin, Ning Xu, Jiang Li, and Liang Qiu performed the experiments. Ju Yang, Maoying Fu, Weifei Fan, Xiaolin $\mathrm{Pu}$, Zhijun $\mathrm{Ge}$, and Feng Zhan collected the data. Ju Yang, Maoying $\mathrm{Fu}$, Jiang Li, and Liang Qiu analyzed the data. Xuefeng $\mathrm{Gu}$ wrote the paper. Jun Wang and Xuefeng $\mathrm{Gu}$ supervised the study. Ju Yang and Maoying Fu contributed equally to this work.

\section{Acknowledgments}

This work was supported by a grant from Natural Science Foundation of Jiangsu Province (Grant no. BK20130895) and Natural Science Research Program for Colleges and Universities in Jiangsu Province (Grant no. 11KJB320006).

\section{References}

[1] C.-W. Huang, H.-L. Tsai, Y.-T. Chen et al., "The prognostic values of EGFR expression and KRAS mutation in patients with synchronous or metachronous metastatic colorectal cancer," BMC Cancer, vol. 13, article 599, 2013.
[2] M. Yasunaga and Y. Matsumura, "Role of SLC6A6 in promoting the survival and multidrug resistance of colorectal cancer," Scientific Reports, vol. 4, article 4852, 2014.

[3] T.-W. Ke, H.-L. Hsu, Y.-H. Wu, W. T.-L. Chen, Y.-W. Cheng, and C.-W. Cheng, "MicroRNA-224 suppresses colorectal cancer cell migration by targeting Cdc42," Disease Markers, vol. 2014, Article ID 617150, 11 pages, 2014.

[4] F.-T. Duan, F. Qian, K. Fang, K.-Y. Lin, W.-T. Wang, and Y.-Q. Chen, "miR-133b, a muscle-specific microRNA, is a novel prognostic marker that participates in the progression of human colorectal cancer via regulation of CXCR4 expression," Molecular Cancer, vol. 12, no. 1, article 164, 2013.

[5] S.-C. Zhang, W. Jin, H. Liu et al., "RPSA gene mutants associated with risk of colorectal cancer among the Chinese population," Asian Pacific Journal of Cancer Prevention, vol. 14, no. 12, pp. 7127-7131, 2013.

[6] M. C. S. Wong, J. Y. L. Ching, H. H. Hirai et al., "Perceived obstacles of colorectal cancer screening and their associated factors among 10,078 Chinese participants," PLoS ONE, vol. 8, no. 7, Article ID e70209, 2013.

[7] X. Lin, Z. Yi, J. Diao et al., "ShaoYao decoction ameliorates colitis-associated colorectal cancer by downregulating proinflammatory cytokines and promoting epithelial-mesenchymal transition," Journal of Translational Medicine, vol. 12, no. 1, article 105, 2014.

[8] W. S. Atkin, R. Edwards, I. Kralj-Hans et al., "Once-only flexible sigmoidoscopy screening in prevention of colorectal cancer: a multicentre randomised controlled trial," The Lancet, vol. 375, no. 9726, pp. 1624-1633, 2010.

[9] Y.-F. Yang, X.-Y. Zhang, M. Yang et al., "Prognostic role of nucleophosmin in colorectal carcinomas," Asian Pacific Journal of Cancer Prevention, vol. 15, no. 5, pp. 2021-2026, 2014.

[10] Y. Li, J. Wei, C. Xu, Z. Zhao, and T. You, "Prognostic significance of cyclin D1 expression in colorectal cancer: a meta-analysis of observational studies," PLoS ONE, vol. 9, no. 4, Article ID e94508, 2014.

[11] W.-H. Leung and S. Bolland, “The inositol 5'-phosphatase SHIP-2 negatively regulates IgE-induced mast cell degranulation and cytokine production," The Journal of Immunology, vol. 179, no. 1, pp. 95-102, 2007.

[12] N. K. Prasad, M. Tandon, S. Badve, P. W. Snyder, and H. Nakshatri, "Phosphoinositol phosphatase SHIP2 promotes cancer development and metastasis coupled with alterations in EGF receptor turnover," Carcinogenesis, vol. 29, no. 1, pp. 25-34, 2008.

[13] A. Koch, A. Mancini, O. El Bounkari, and T. Tamura, "The SH2domian-containing inositol 5-phosphatase (SHIP)-2 binds to cMet directly via tyrosine residue 1356 and involves hepatocyte growth factor (HGF)-induced lamellipodium formation, cell scattering and cell spreading," Oncogene, vol. 24, no. 21, pp. 3436-3447, 2005.

[14] S. Sumie, T. Kawaguchi, M. Komuta et al., "Significance of glucose intolerance and SHIP2 expression in hepatocellular carcinoma patients with HCV infection," Oncology Reports, vol. 18, no. 3, pp. 545-552, 2007.

[15] X. B. Xu, J. Qin, and W. Y. Liu, "Curcumin inhibits the invasion of thyroid cancer cells via down-regulation of PI3K/Akt signaling pathway," Gene, vol. 546, no. 2, pp. 226-232, 2014.

[16] J. J. Wheler, S. L. Moulder, A. Naing et al., "Anastrozole and everolimus in advanced gynecologic and breast malignancies: activity and molecular alterations in the $\mathrm{PI} 3 \mathrm{~K} / \mathrm{AKT} / \mathrm{mTOR}$ pathway," Oncotarget, vol. 5, no. 10, pp. 3029-3038, 2014. 
[17] S. Giuriato, D. Blero, B. Robaye, C. Bruyns, B. Payrastre, and C. Erneux, "SHIP2 overexpression strongly reduces the proliferation rate of K562 erythroleukemia cell line," Biochemical and Biophysical Research Communications, vol. 296, no. 1, pp. 106110, 2002.

[18] T. Sasaoka, K. Kikuchi, T. Wada et al., "Dual role of SRC homology domain 2-containing inositol phosphatase 2 in the regulation of platelet-derived growth factor and insulin-like growth factor I signaling in rat vascular smooth muscle cells," Endocrinology, vol. 144, no. 9, pp. 4204-4214, 2003.

[19] N. K. Prasad, M. Tandon, A. Handa et al., "High expression of obesity-linked phosphatase SHIP2 in invasive breast cancer correlates with reduced disease-free survival," Tumor Biology, vol. 29, no. 5, pp. 330-341, 2008.

[20] X. Zhou, Y. Liu, and G. Tan, "Prognostic value of elevated SHIP2 expression in laryngeal squamous cell carcinoma," Archives of Medical Research, vol. 42, no. 7, pp. 589-595, 2011.

[21] N. K. Prasad, "SHIP2 phosphoinositol phosphatase positively regulates EGFR-Akt pathway, CXCR4 expression, and cell migration in MDA-MB-231 breast cancer cells," International Journal of Oncology, vol. 34, no. 1, pp. 97-105, 2009.

[22] F. Obrocea, M. Sajin, E. C. Marinescu, and D. Stoica, "Colorectal cancer and the 7th revision of the TNM staging system: review of changes and suggestions for uniform pathologic reporting," Romanian Journal of Morphology and Embryology, vol. 52, no. 2, pp. 537-544, 2011.

[23] M. Fu, W. Fan, X. Pu et al., "Elevated expression of SHIP2 correlates with poor prognosis in non-small cell lung cancer," International Journal of Clinical and Experimental Pathology, vol. 6, no. 10, pp. 2185-2191, 2013.

[24] M. Fu, X. Gu, H. Ni et al., "High expression of inositol polyphosphate phosphatase-like 1 associates with unfavorable survival in hepatocellular carcinoma," International Journal of Clinical and Experimental Pathology, vol. 6, no. 11, pp. 25152522, 2013.

[25] V. Taylor, M. Wong, C. Brandts et al., " 5 ' Phospholipid phosphatase SHIP-2 causes protein kinase B inactivation and cell cycle arrest in glioblastoma cells," Molecular \& Cellular Biology, vol. 20, no. 18, pp. 6860-6871, 2000.

[26] A. Suwa, T. Kurama, and T. Shimokawa, "SHIP2 and its involvement in various diseases," Expert Opinion on Therapeutic Targets, vol. 14, no. 7, pp. 727-737, 2010.

[27] B. Ducarouge, M. Pelissier-Rota, M. Lainé et al., "CRF2 signaling is a novel regulator of cellular adhesion and migration in colorectal cancer cells," PLoS ONE, vol. 8, no. 11, Article ID e79335, 2013.

[28] N. Vassos, T. Rau, S. Merkel et al., "Prognostic value of $\beta 1$ integrin expression in colorectal liver metastases," International Journal of Clinical and Experimental Pathology, vol. 7, no. 1, pp. 288-300, 2014.

[29] J. F. Groulx, V. Giroux, M. Beausejour et al., "Integrin alpha6A splice variant regulates proliferation and the Wnt/beta-catenin pathway in human colorectal cancer cells," Carcinogenesis, vol. 35, no. 6, pp. 1217-1227, 2014.

[30] J. M. Haugh, F. Codazzi, M. Teruel, and T. Meyer, "Spatial sensing in fibroblasts mediated by $3^{\prime}$ phosphoinositides," The Journal of Cell Biology, vol. 151, no. 6, pp. 1269-1280, 2000.

[31] A. Y. Alexandrova, "Evolution of cell interactions with extracellular matrix during carcinogenesis," Biochemistry, vol. 73, no. 7, pp. 733-741, 2008. 


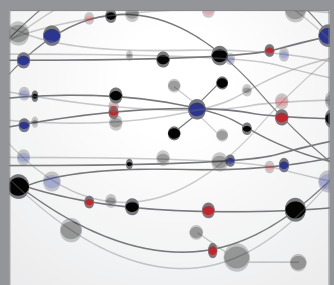

The Scientific World Journal
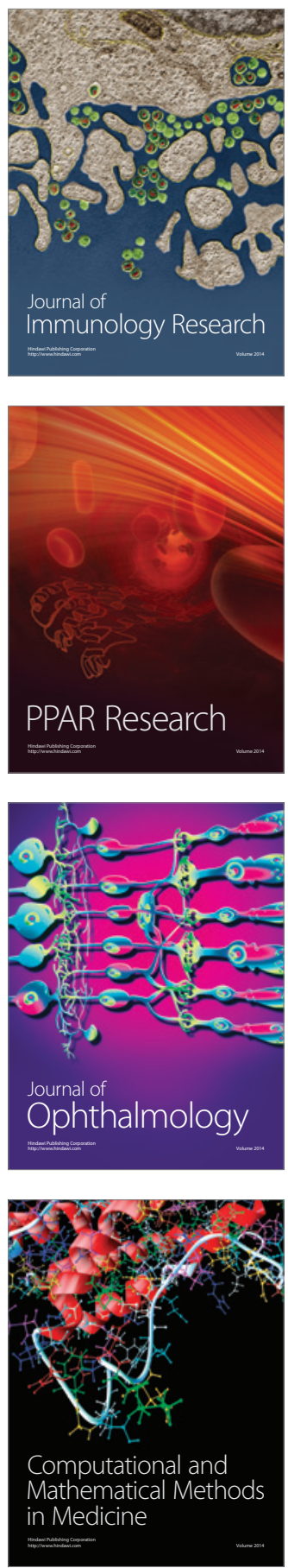

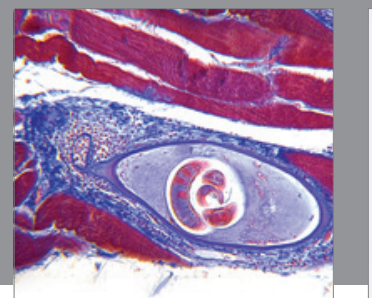

Gastroenterology

Research and Practice
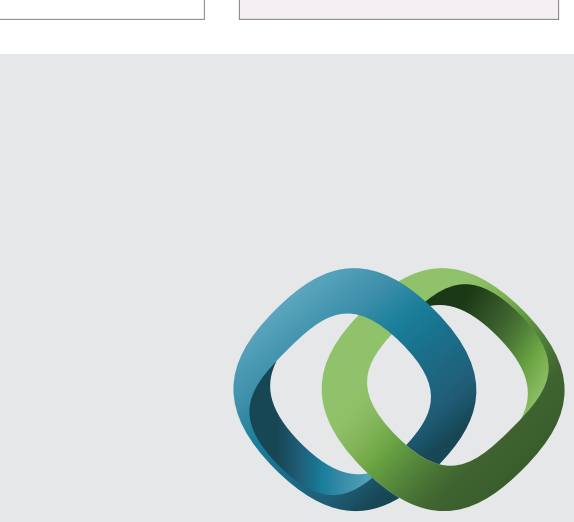

\section{Hindawi}

Submit your manuscripts at

http://www.hindawi.com
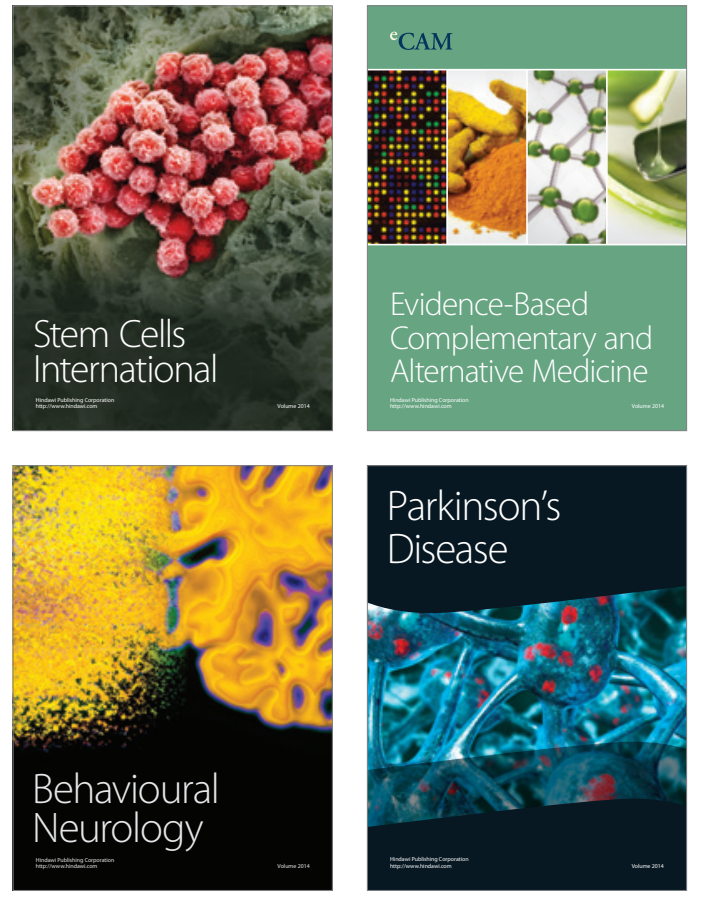
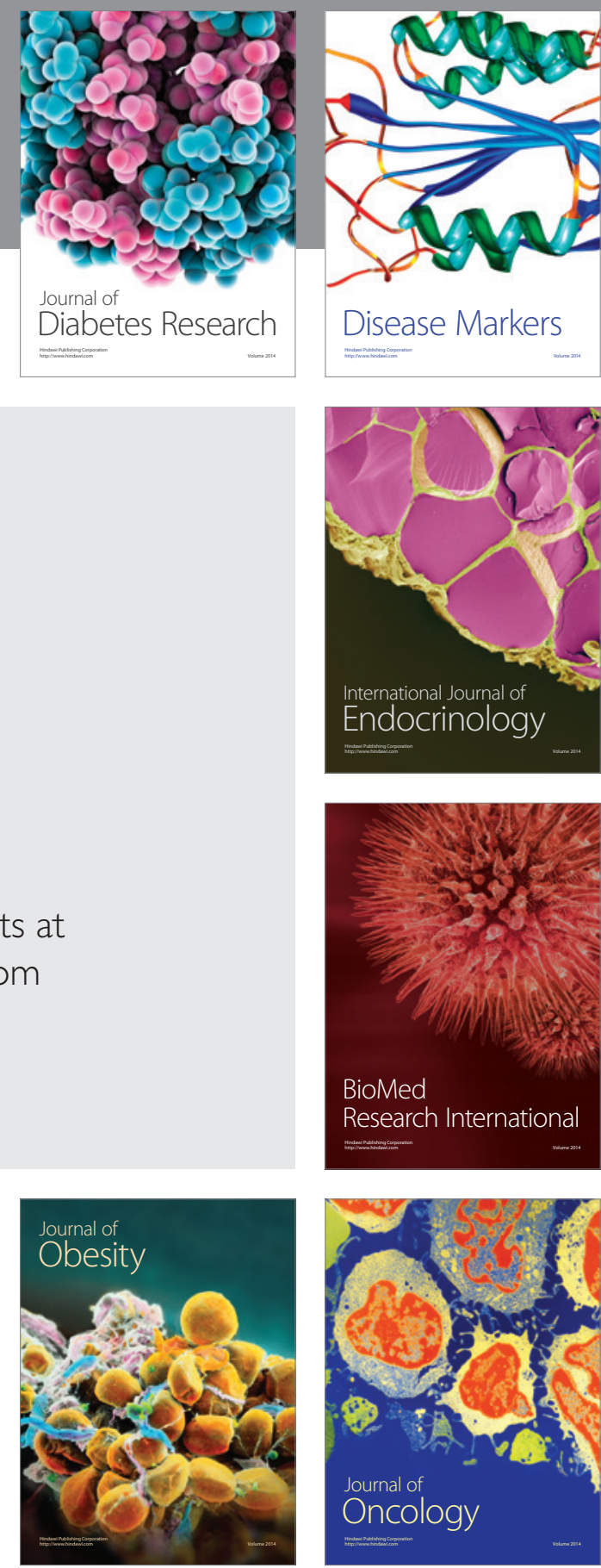

Disease Markers
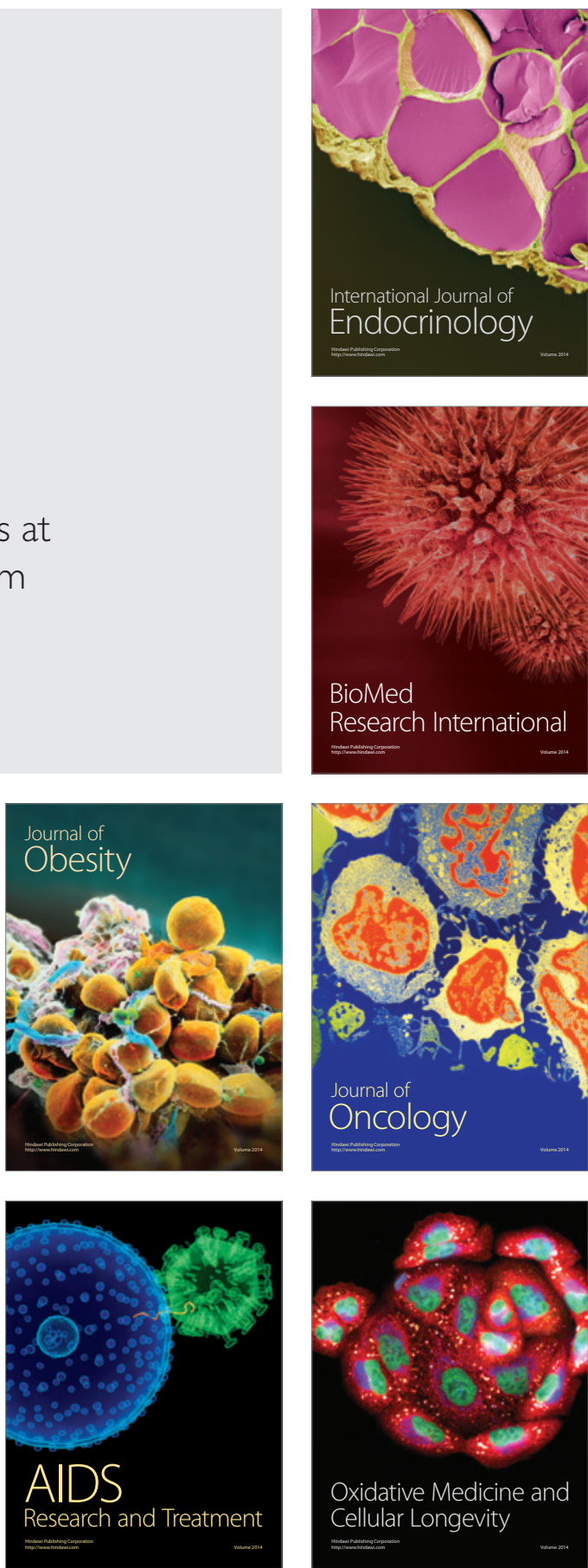